\title{
Maximum $N$-body correlations do not in general imply genuine multipartite entanglement
}

\author{
Christopher Eltschka ${ }^{1}$ and Jens Siewert ${ }^{2,3}$ \\ ${ }^{1}$ Institut für Theoretische Physik, Universität Regensburg, D-93040 Regensburg, Germany \\ ${ }^{2}$ Departamento de Química Física, Universidad del País Vasco UPV/EHU, E-48080 Bilbao, Spain \\ ${ }^{3}$ IKERBASQUE Basque Foundation for Science, E-48013 Bilbao, Spain
}

The existence of correlations between the parts of a quantum system on the one hand, and entanglement between them on the other, are different properties. Yet, one intuitively would identify strong $N$-party correlations with $N$-party entanglement in an $N$-partite quantum state. If the local systems are qubits, this intuition is confirmed: The state with the strongest $N$-party correlations is the Greenberger-Horne-Zeilinger (GHZ) state, which does have genuine multipartite entanglement. However, for high-dimensional local systems the state with strongest $N$-party correlations may be a tensor product of Bell states, that is, partially separable. We show this by introducing several novel tools for handling the Bloch representation.

\section{Introduction}

The expansion of the density operator in terms of a matrix basis is called the Bloch representation [1, 2, 3]. Technically, this representation is rather demanding: A pure state of $N$ parties each of local dimension $d$ is characterized by $2 d^{N}-2$ real coefficients, whereas the same state written in the Bloch representation requires $d^{2 N}-1$ parameters, just as any mixed state. On the other hand, this representation appears to be perfectly adapted to studying the correlation properties of a quantum system, because the above-mentioned expansion corresponds to a decomposition of the state into all possible correlation contributions (hence the terms are also called correlation tensors). Therefore, from a better understanding of the technical characteristics of this expansion one may expect significant insight into the physics of correlated quantum systems.

The systematic investigation of the properties of the Bloch representation for finite-dimensional multi-party quantum systems is a relatively recent subject $[4,5,6,7,8,9,10,11,12,13$, 14, 15], although many important results were found earlier, mostly relating specific features of the Bloch picture to the entanglement properties of the state (e.g., [16, 17, 18, 19, 20, 21, 22, 23, 24, 26, 25]). Currently much activity is devoted to working out the technical details and properties for an easier use of the Bloch representation to solving physics problems. An essential part of this is to figure out smaller sets of parameters that carry sufficient amounts of information to facilitate the characterization of relevant physical properties for a state given in the Bloch representation. In this contribution, we define such a set of parameters, which we call the "sector distribution" and discuss some of its key features. Moreover, we illustrate how the properties of this distribution are reflected in the correlation properties of the states.

To be more specific, let us preliminarily introduce the Bloch representation; the precise definition will be given below. If we enumerate the parties of an $N$-party system (of equal local dimension $d$ ) by $\{1,2 \ldots N\}$ and $A$ is a subset of parties, then

$$
\rho=\frac{1}{d^{N}} \sum_{A} G_{A} \otimes \mathbb{1}_{\bar{A}}
$$

Here, $G_{A}$ is a Hermitian operator that acts nontrivially on the parties belonging to the subset $A$, and $\mathbb{1}_{\bar{A}}$ is the identity operator for the complementary set. Consider now the sum of all those terms in Eq. (1) that act on the same 
number $k$ of parties,

$$
\mathcal{S}_{k} \equiv \frac{1}{d^{N}} \sum_{|A|=k} G_{A} \otimes \mathbb{1}_{\bar{A}}
$$

We call $\mathcal{S}_{k}$ the " $k$-sector" of $\rho$ and the (squared) Hilbert-Schmidt length of $\mathcal{S}_{k}$ the " $k$-sector length" $S_{k}[27]$

$$
S_{k} \equiv d^{N} \operatorname{Tr}\left(\mathcal{S}_{k}^{\dagger} \mathcal{S}_{k}\right)
$$

The $k$-sector length $S_{k}$ is a natural quantifier for the $k$-party correlations in a state [28]. Clearly, for an $N$-partite state there are $N$ sector lengths $\left(\mathcal{S}_{0}=1\right.$ for all normalized states). Sector lengths were discussed earlier $[16,17,20,4$, $5,6,8,7,9,10,14,15]$. In particular the $N$-sector was intuitively linked with the $N$-party quantum correlations. Therefore it came as a surprise that there exist mixed states that are $N$-party entangled but do not possess $N$-party correlations [29, 30, 31, 32]. Later it was realized [4] that, in order to witness genuine multipartite entanglement, it may be necessary to consider a collection of the highest sector lengths $S_{N}, S_{N-1}, \ldots$ rather than just $S_{N}$. In the present work we systematically study the set of all sector lengths $\left\{S_{k}\right\}$. As we will demonstrate the distribution $\left\{S_{1}, S_{2} \ldots S_{N}\right\}$ represents a reduced set of parameters in the spirit described above (linear in the system size instead of exponential) that carries substantial information regarding some of the correlation properties of the state. Often it has little meaning to study the sector lengths $S_{k}$ individually; rather, there exists a variety of strict relations between them that determine the entire distribution.

We introduce several novel technical concepts, most importantly the $N$-sector projector. We use this toolbox to prove the long-standing conjecture that for any number $N$ of qubits the GHZ state maximizes the $N$-sector length. Subsequently we analyze the sector distribution for few-party systems of higher local dimension $d>2$. Here we prove that for higher local dimension the state with maximum $N$-sector length may be partially separable. Moreover, we provide a comprehensive discussion for the behavior of the $N$-sector with increasing number of parties as well as growing local dimension.

\section{Definitions and preliminaries}

For the Bloch representation of an $N$-partite state with all local dimensions equal to $d \geqq 2$ the operators $G_{A}$ in Eq. (1) are expanded in local operators, i.e., a basis of traceless matrices $\left\{\mathrm{g}_{j}\right\}$, $1 \leqq j \leqq d^{2}-1, \mathrm{~g}_{0} \equiv \mathbb{1}$, with normalization $\operatorname{Tr}\left(\mathrm{g}_{j}^{\dagger} \mathrm{g}_{k}\right)=d \delta_{j k}$,

$$
\rho=\frac{1}{d^{N}} \sum_{A} \sum_{j_{l}: l \in A} r_{j_{1} \cdots j_{N}} \mathrm{~g}_{j_{1}} \otimes \cdots \otimes \mathrm{g}_{j_{N}} \otimes \mathbb{1}_{\bar{A}},
$$

and

$$
r_{j_{1} \cdots j_{N}}=\operatorname{Tr}\left(\left[\mathrm{g}_{j_{1}}^{\dagger} \otimes \cdots \otimes \mathrm{g}_{j_{N}}^{\dagger} \otimes \mathbb{1}_{\bar{A}}\right] \rho\right) .
$$

Here, all indices $j_{m}, m \in \bar{A}$ are set to 0 . With this, the $k$-sector length simply becomes

$$
S_{k}=\sum_{j_{l}: l \in A,|A|=k}\left|r_{j_{1} \cdots j_{N}}\right|^{2},
$$

where $|A|$ is the number of elements in $A$. All the sector lengths are local unitary invariants of the state. As the actual sector lengths $\sqrt{S_{k}}$ equal the Hilbert-Schmidt norms of $\mathcal{S}_{k}$, they obey the triangle inequality. Because of this, the $N$-sector length of a mixture of pure states can never exceed the largest $N$-sector length of any of the pure states. Therefore, throughout this article we focus on pure states $\Pi$, that is, for the purity we have $\operatorname{Tr} \Pi^{2}=\operatorname{Tr} \Pi=1$ and, hence, for the sum of all sector lengths, $\sum_{k=0}^{N} S_{k}=d^{N}$.

Consider the simplest case of all, that is, product states $\left|\operatorname{prod}_{j}^{N}\right\rangle=|j\rangle^{\otimes N}$; here $|j\rangle$ denotes a state of the computational basis, $j=0,1, \ldots,(d-1)$. It is easy to see that $S_{k}\left(\operatorname{prod}_{j}^{N}\right)=\left(\begin{array}{c}N \\ k\end{array}\right)(d-1)^{k}$. Remarkably, it was shown by Tran et al. [6] that among the pure states only product states have the minimum $N$-sector length

$$
\min S_{N}=(d-1)^{N},
$$

that is, for $d>2$ the $N$-sector is always on the order of $d^{N}$. It turned out that the opposite question regarding the states with maximum $N$-sector is considerably more complex. A relevant state for this discussion is the GHZ state for $N$ parties of local dimension $d$

$$
\left|\mathrm{GHZ}_{d}^{N}\right\rangle=\frac{1}{\sqrt{d}} \sum_{j=0}^{d-1}|j\rangle^{\otimes N} .
$$


For $N$-qudit GHZ states we find the sector distribution (see Appendix)

$$
\begin{aligned}
S_{k}\left(\mathrm{GHZ}_{d}^{N}\right)= & (d-1) d^{N-1} \delta_{k N}+ \\
& +\left(\begin{array}{c}
N \\
k
\end{array}\right) \frac{(d-1)^{k}+(-1)^{k}(d-1)}{d} .
\end{aligned}
$$

Tran et al. [6] showed that for odd party number $N$ of qubits the GHZ state has maximum $N$-sector length. They conjectured that this statement holds also for even $N$. For $d>2$ it is not clear which state has maximum $N$-party correlations. In the following we will prove the conjecture for even- $N$ qubit GHZ states.

\section{The $N$-sector projector}

For the proof we need some mathematical tools based on universal state inversion [35, 36, 37, 9, 12]. First we define the projection (super-)operator [38] onto the last $(k=N)$ sector (or $N$-sector projector for short whenever there are no ambiguities)

$$
\mathcal{P}(\rho)=\prod_{j=1}^{N}\left[\text { id }-\frac{1}{d} \operatorname{Tr}_{j}(\cdot) \otimes \mathbb{1}_{j}\right] \rho .
$$

It is easy to check by writing $\rho$ in the Bloch representation that $\mathcal{P}$ indeed realizes a projection onto the $N$-sector, $\mathcal{S}_{N}$. The map (9) belongs to the class of generalized universal state inversions discussed in Refs. [37, 12] that have the form $\mathcal{I}^{\left\{\alpha_{j}, \beta_{j}\right\}}=\prod_{j=1}^{N}\left[\alpha_{j} \operatorname{Tr}_{j}(\cdot) \otimes \mathbb{1}_{j}-\beta_{j}\right.$ id $]$, where id denotes the identity map and $\alpha_{j}, \beta_{j}$ are real numbers. With definition (9) we get immediately

$$
\begin{aligned}
S_{N}(\rho) & =d^{N} \operatorname{Tr}[\rho \mathcal{P}(\rho)] \\
& =d^{N} \sum_{A}\left(-\frac{1}{d}\right)^{|A|} \operatorname{Tr}\left(\rho_{\bar{A}}^{2}\right),
\end{aligned}
$$

where $A$, as before, runs through all subsets of $\{1 \ldots N\}$ and $\rho_{\bar{A}}=\operatorname{Tr}_{A} \rho$ is the reduced state on the subset of parties $\bar{A}$. The equality
$\operatorname{Tr}\left[\left(\operatorname{Tr}_{A} \Pi\right)^{2}\right]=\operatorname{Tr}\left[\left(\operatorname{Tr}_{\bar{A}} \Pi\right)^{2}\right]$ for pure states $\Pi$ motivates the definition of another operator

$$
\mathcal{Q}(\rho)=\prod_{j=1}^{N}\left[\operatorname{Tr}_{j}(\cdot) \otimes \mathbb{1}_{j}-\frac{1}{d} \mathrm{id}\right] \rho,
$$

so that

$$
\begin{aligned}
S_{N} & =d^{N} \operatorname{Tr}[\Pi \mathcal{P}(\Pi)] \\
& =d^{N} \operatorname{Tr}[\Pi \mathcal{Q}(\Pi)] \geqq 0
\end{aligned}
$$

Because of the projector property of $\mathcal{P}$ and the Cauchy-Schwarz inequality, $\|M\|_{\mathcal{P}} \equiv$ $\sqrt{\operatorname{Tr}\left[M^{\dagger} \mathcal{P}(M)\right]}$ defines a seminorm for operators $M$ (while the analogous statement does not hold for $\mathcal{Q})$. By considering the action of $\mathcal{Q}(\Pi)$ in the Bloch representation relation (12) gives rise to the astounding equality

$$
d^{N} S_{N}(\Pi)=\sum_{k=0}^{N}(-1)^{k}\left(d^{2}-1\right)^{N-k} S_{k}(\Pi),
$$

which links the last sector length $S_{N}$ with all others.

Finally we rewrite the well-known purity condition for reductions of pure states $\operatorname{Tr}\left[\left(\operatorname{Tr}_{A}|\psi\rangle\langle\psi|\right)^{2}\right]=\operatorname{Tr}\left[\left(\operatorname{Tr}_{\bar{A}}|\psi\rangle\langle\psi|\right)^{2}\right] \quad$ in terms of sector lengths. This is achieved by symmetrizing the purity conditions for fixed $|A|$ and accomplishing the combinatorial accounting. We find for the $k$-purity relation $\left(k=0,1, \ldots,\left\lfloor\frac{N-1}{2}\right\rfloor\right.$ with the floor function $\lfloor\cdot\rfloor)[33]$

$$
d^{N-2 k} \sum_{m=0}^{k}\left(\begin{array}{c}
N-m \\
k-m
\end{array}\right) S_{m}=\sum_{n=0}^{N-k}\left(\begin{array}{c}
N-n \\
k
\end{array}\right) S_{n} .
$$

For $k=0$ this gives the well-known condition $d^{N}=\sum_{0}^{N} S_{n}$. We explicitly write the relations for $k=1$ and $k=2$ as they will turn out useful later:

$$
\begin{aligned}
d^{N-2}\left[N+S_{1}\right] & =N+(N-1) S_{1}+\ldots+2 S_{N-2}+S_{N-1} \\
d^{N-4}\left[\left(\begin{array}{c}
N \\
2
\end{array}\right)+(N-1) S_{1}+S_{2}\right] & =\left(\begin{array}{c}
N \\
2
\end{array}\right)+\left(\begin{array}{c}
N-1 \\
2
\end{array}\right) S_{1}+\ldots+\left(\begin{array}{l}
3 \\
2
\end{array}\right) S_{N-3}+S_{N-2}
\end{aligned}
$$


Interestingly, Eqs. (14) elucidate the role of the $N$-sector for pure states: All sector lengths have to be adjusted so as to obey the $k$-purity relations $\left(k=1 \ldots\left\lfloor\frac{N-1}{2}\right\rfloor\right)$ between the reduced states of non-empty complementary partitions; note that the last sector is excluded from establishing this balance. The last sector serves to fill up the total length $d^{N}$ of the Bloch vector (the "0-purity" relation).

\section{The $N$-qubit $\mathrm{GHZ}$ state maximizes the $N$-sector}

For odd $N$ we know $\max S_{N}=S_{N}(\mathrm{GHZ})=$ $2^{N-1}$, cf. Ref. [6]. We recall this proof in the Appendix. We are prepared now to show for even $N$ qubits that

$$
\max S_{N}=S_{N}(\mathrm{GHZ})=2^{N-1}+1 .
$$

As here the GHZ state has only even-numbered sectors, Eq. (13) would imply for a hypothetical state $\Pi_{x}=|x\rangle\langle x|$ with larger $N$-sector than GHZ that $S_{2 m}>\left(\begin{array}{c}N \\ 2 m\end{array}\right)$ for some $m<N / 2$. In order to obtain information regarding the distribution of the even-numbered sectors we consider an $R$ matrix (analogous to Refs. [34, 5, 9]) of $\rho_{[1]} \equiv$ $\operatorname{Tr}_{\{1\}} \Pi_{x}$ after tracing the first party,

$$
R_{[1]} \equiv \rho_{[1]} \mathcal{I}_{-}\left(\rho_{[1]}\right)=\rho_{[1]} \sum_{A}(-1)^{|A|} \operatorname{Tr}_{\bar{A}} \rho_{[1]} \otimes \mathbb{1}_{\bar{A}}
$$

where $\mathcal{I}_{-}(\sigma) \equiv \prod_{j=1}^{M}\left[\operatorname{Tr}_{j}(\cdot) \otimes \mathbb{1}_{j}-\right.$ id $] \sigma$ denotes the standard universal state inversion for an $M$-partite state $\sigma$ (cf. Refs. [36, 9, 11]). If we symmetrize over the traced party we can establish a relation between $\sum_{j} \operatorname{Tr} R_{[j]}$ and the sector lengths $S_{k}\left(\Pi_{x}\right)$ of $\Pi_{x}$,

$0 \leqq d^{N-1} \sum_{j=1}^{N} \operatorname{Tr} R_{[j]}=\sum_{k=0}^{N-1}(-1)^{k}(N-k) S_{k}$.

The reasoning is exactly the same as the one to obtain the 1-purity, Eq. (15a). By adding the latter equation and Eq. (17) (and dividing by 2) we obtain a relation for the even-numbered sectors,

$$
\frac{d^{N-2}}{2}\left[N+S_{1}+\sum_{j} d \operatorname{Tr} R_{[j]}\right]=N+(N-2) S_{2}+(N-4) S_{4}+\ldots+4 S_{N-4}+2 S_{N-2} .
$$

We observe that on the right-hand side (r.h.s.) the prefactors increase with decreasing index, this is analogous to Eq. (13), only that here the prefactors increase linearly. Also here the even-sector distribution of $\Pi_{x}$ would exceed the result of the GHZ state. Relation (18) gives us the possibility to directly check the achievable maximum of the r.h.s. for pure states by maximizing the terms on the left-hand side. For qubits this is straightforward and shows that the maximum is achieved for the GHZ state (we present this calculation in the Appendix). Hence, there is no state $\Pi_{x}$ with larger $N$-sector.

\section{Few parties of higher local dimension}

The obvious guess from the results so far is that $\left|\mathrm{GHZ}_{d}^{N}\right\rangle$ maximizes the $N$-sector length also for $d>2$. It will turn out that this can only partially be true. To this end, let us investigate states with up to six parties. The following results are obtained by using Eqs. (13), (14) for $k=0,1,2$, and increasingly tedious algebra.

$N=2:$ We have $d^{2}=1+S_{1}+S_{2}$, so that

$$
\max S_{2}=d^{2}-1
$$

for $S_{1}=0$, that is, the Bell state $\left|\Phi_{d}^{+}\right\rangle \equiv\left|\mathrm{GHZ}_{d}^{2}\right\rangle$ maximizes the 2 -sector.

$N=3$ : Here,

$$
S_{3}=(d-1)^{2}(d+2)-(d-1) S_{1},
$$


so that $S_{1}=0$ leads to $\max S_{3}=(d+2)(d-1)^{2}$, which again is realized by the GHZ state.

$N=4$ : In this case, there remains more than one parameter undetermined

$$
S_{4}=\left(d^{2}-1\right)^{2}-\frac{1}{2}\left[\left(d^{2}-1\right) S_{1}+S_{3}\right],
$$

but since $S_{1}, S_{3} \geqq 0$, the 4 -sector gets maximized for $S_{1}=S_{3}=0$, so that $\max S_{4}=\left(d^{2}-1\right)^{2}$. That is, for four-party states the $N$-sector is not maximized by the GHZ state, but by a tensor product of Bell states, i.e., a biseparable state. Curiously, the case $d=2$ is right on the edge, because the tensor product of a pair of two-qubit Bell states and the four-qubit GHZ state have the same 4-sector length, $S_{4}=9$.

$N=5$ : Here we find

$$
\begin{aligned}
(d-3) S_{5} & =(d-1)^{3}(d+2)\left(d^{2}-2 d-4\right)- \\
& -(d-1)^{2}\left(d^{2}-d-3\right) S_{1}+(d-1) S_{3} .
\end{aligned}
$$

This suggests again $S_{1}=0$ for the $N$-sector maximum, however, now the sign of $S_{3}$ is reversed. We note that the maximum $k$-sector length of an $N$-party system is on the order of $\left(\begin{array}{c}N \\ k\end{array}\right) d^{k}$, so that the 3-sector length $S_{3} \sim O\left(d^{3}\right)$, and hence for large local dimension $d \gg 1$

$$
\frac{\max S_{5}}{(d-1)^{3}(d+1)(d+2)} \longrightarrow 1+O\left(d^{-2}\right),
$$

which indicates that the maximum $N$-sector is approximated with better than first-order accuracy for growing $d$ by the polynomial in the denominator. The latter corresponds to the tensor product of a Bell state and a three-party GHZ state, $\left|\Phi_{d}^{+}\right\rangle \otimes\left|\mathrm{GHZ}_{d}^{3}\right\rangle$. Consequently, for large $d$ also here the state with maximum $N$-body correlations may be biseparable. The case $d=3$ is special: $S_{3}=20$ gives the largest 3 -sector. The five-qutrit GHZ state is compatible with this [cf. Eq. (8)] and has larger 5-sector than $\left|\Phi_{3}^{+}\right\rangle \otimes\left|\mathrm{GHZ}_{3}^{3}\right\rangle$ (172 vs. 160). However, in principle, there might be a state with $S_{1}=0$, $S_{3}=20$ and even larger 5-sector.

$N=6$ : This case has similar features as $N=5$. The 6-sector obeys

$$
\begin{aligned}
2\left(d^{2}-4\right) S_{6}= & 2(d-2)\left(d^{2}-1\right)^{3}(d+2)- \\
& -\left(d^{2}-1\right)^{2}\left(d^{2}-3\right) S_{1}+ \\
& +\left(d^{2}-1\right) S_{3}-\left(d^{2}-3\right) S_{5} .
\end{aligned}
$$

Again we see that for increasing $d \gg 1$ the 6-sector $\left(\max S_{6}\right) /\left(d^{2}-1\right)^{3} \longrightarrow 1+O\left(d^{-3}\right)$ because of the scaling of the sector lengths with $d$; the corresponding state is $\left|\Phi_{d}^{+}\right\rangle^{\otimes 3}$. Note that already for $d=3$ the 6 -sector of $\left|\Phi_{3}^{+}\right\rangle^{\otimes 3}$ beats the length of the six-qutrit GHZ state (512 vs. 508).

We summarize the results of this section in Tables 1-3.

Table 1: Maximum of the $N$-sector for 2, 3, 4 parties.

\begin{tabular}{ccc}
\hline \hline$N$ & $\max N$-sector $S_{N}$ & state maximizing $S_{N}$ \\
\hline 2 & $d^{2}-1$ & $\left|\Phi_{d}^{+}\right\rangle$ \\
3 & $(d-1)^{2}(d+2)$ & $\left|\mathrm{GHZ}_{d}^{3}\right\rangle$ \\
4 & $\left(d^{2}-1\right)^{2}$ & $\left|\Phi_{d}^{+}\right\rangle^{\otimes 2}$ \\
\hline \hline
\end{tabular}

Table 2: Comparison of $N$-sectors for $N=5$.

\begin{tabular}{c|ccc}
\hline \hline$S_{5}$ & $\begin{array}{c}\left|\mathrm{GHZ}_{d}^{5}\right\rangle \\
(d-1)^{2} \times\end{array}$ & & $\begin{array}{c}\left.\mathrm{GHZ}_{d}^{3}\right\rangle \otimes\left|\Phi_{d}^{+}\right\rangle \\
(d-1)^{3} \times\end{array}$ \\
& $\left(d^{3}+2 d^{2}-2 d+4\right)$ & & $(d+1)(d+2)$ \\
\hline 3 & 172 & $>$ & 160 \\
4 & 828 & $>$ & 810 \\
5 & 2704 & $>$ & 2688 \\
6 & 7000 & $=$ & 7000 \\
7 & 15516 & $<$ & 15552 \\
\hline \hline
\end{tabular}

Table 3: Comparison of $N$-sectors for $N=6$.

\begin{tabular}{cccc}
\hline \hline$S_{6}$ & $\left|\mathrm{GHZ}_{d}^{6}\right\rangle$ & & $\left|\Phi_{d}^{+}\right\rangle^{\otimes 3}$ \\
$d$ & $\frac{d-1}{d}\left[d^{6}+(d-1)^{5}+1\right]$ & & $\left(d^{2}-1\right)^{3}$ \\
\hline 2 & 33 & $>$ & 27 \\
3 & 508 & $<$ & 512 \\
4 & 3255 & $<$ & 3375 \\
\hline \hline
\end{tabular}

\section{Maximum $N$-sector for large $d$ and large $N$}

We can investigate the dominance of $N$-sectors numerically. On increasing $d$, the partially separable states - that is, a tensor product of Bell states (even $N$ ) or Bell states and a three-party 

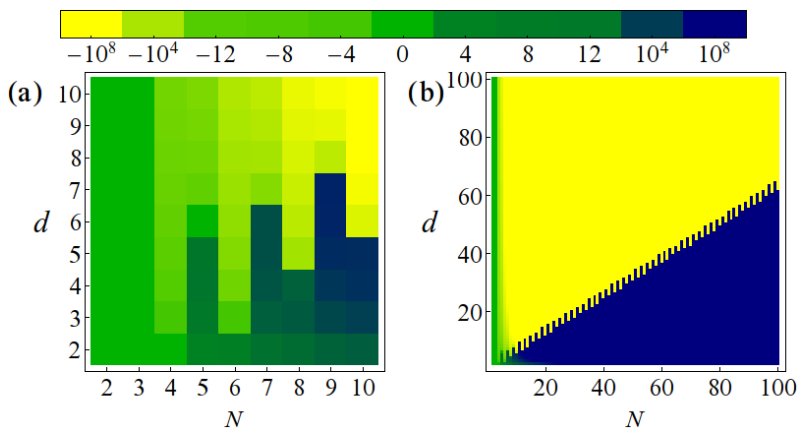

Figure 1: $N$-sector length difference $S_{N}\left(\mathrm{GHZ}_{d}^{N}\right)-S_{N}\left(\mathrm{Bell}_{d}^{N}\right)$. The border between GHZ-dominated and Bell-dominated is given by a straight line $d \simeq 0.6275 \cdot N$ (see text); however, note the pronounced even-odd effect. For $N=2$ and $N=3$, $\mathrm{GHZ}$ and Bell are the same state, therefore these cases have to be counted as 'undecided'. (a) Small scale $N, d \leqq 10$; (b) larger scale $N, d \leqq 100$. Note that the color scale is logarithmic.

GHZ state (odd $N$ ) -appear to dominate (we will call these states "Bell"). In the opposite case, the GHZ state has larger $N$-sector. This behavior is shown in Fig. 1, where the difference of $N$-sectors $S_{N}\left(\mathrm{GHZ}_{d}^{N}\right)-S_{N}\left(\right.$ Bell $\left._{d}^{N}\right)$ is displayed in a $(d, N)$ plane. We note the formal analogy of our problem of finding the maximum $N$-sector with that of deciding the existence of absolutely maximimally entangled (AME) states [21, 39, $40,8,11]$ that is suggested by the structure of Fig. 1. The analogy arises because also the $N$-sector problem seems to have two solutions whose validity regions in the $(d, N)$ plane are connected and separated by a single line. The region of GHZ dominance corresponds to 'AME state does not exist', while that of Bell dominance relates to 'AME does exist'. The line separating the two corresponds to the Scott bound [21, 11]. An accurate analytical approximation for this line is found by equating $S_{N}\left(\mathrm{GHZ}_{d}^{N}\right)$ in Eq. (8) with $S_{N}\left(\right.$ Bell $\left._{d}^{N}\right)=\left(d^{2}-1\right)^{N / 2}$ (for even $\left.N\right)$. Assuming $d=\gamma^{-1} N$, this leads to an equation that determines the $\gamma$ parameter, $\mathrm{e}^{-\gamma}=1-\frac{\gamma}{2}$, from which

$$
d \simeq 0.6275 \cdot N \text {. }
$$

Close to this line there may be exceptions from the rule, just as in the case of AME states.

In the following we provide arguments why GHZ and Bell are, if not the dominating, at least close to the states with dominating $N$-sector in the limits of large $N$ and $d$. Consider first fixed even $N$ and $d \gg 2, N$. Our reasoning is based on the purity relations Eqs. (15) and on the consideration that to leading order the maximum $k$-sector is given by $S_{k} \sim\left(\begin{array}{c}N \\ k\end{array}\right) d^{k}$. From the 0-purity relation $S_{N}=d^{N}-S_{N-1}-\ldots-1$ it follows that the dominating terms $S_{N-1}+S_{N-2}$ need to be as small as possible in order to obtain $\max S_{N}$. We observe that Eq. (15a) dictates that $S_{N-2}$ and $S_{N-1}$ cannot both vanish, and their sum needs to be at least of order $N d^{N-2}$. As $S_{1}>0$ would only increase the r.h.s., $S_{1}=0$ is the sensible choice. Moreover, we see that $S_{N-2}+S_{N-1} \approx \frac{1}{2}\left(d^{N-2} N+S_{N-1}\right)$, so that the subleading correction becomes smallest for $S_{N-1}=0$ and $S_{N-2} \approx \frac{N}{2} d^{N-2}$. Substituting this result into Eq. (15b) leads to $S_{2} \approx \frac{N}{2} d^{2}$. In particular the latter requirement together with $S_{1}=0$ can be fulfilled if the state is a Bell tensor product.

For the opposite limit, $N \gg d>2$, general statements are more difficult to make, because the sector sum does not correspond to a power expansion in $d$ any longer. We can discuss at least the case of states that are more entangled than GHZ, that is, $m$-uniform states [21, 39]. A state is called $m$-uniform if $S_{1}=S_{2}=\cdots=S_{m}=0$, with the extreme case of AME states $(m=$ $\lfloor N / 2\rfloor)$. For AME states, $S_{N} \simeq d^{N}\left(1-\frac{1}{d^{2}}\right)^{N}$ is a fair approximation that applies to some extent also to $m \lesssim N / 2$ if $N$ does not exceed $d^{2}$. Then, for large $d$ approximately $S_{N} \sim d^{N} \mathrm{e}^{-\frac{N}{d^{2}}}$, which shows that a substantial fraction of the Bloch vector length is not in the $N$-sector, making these states bad candidates for the maximum $S_{N}$.

On the other hand, for the GHZ state we have

$$
S_{N}\left(\mathrm{GHZ}_{d}^{N}\right) \simeq d^{N}\left(1-\frac{1}{d}\right)+d^{N-1} \mathrm{e}^{-\frac{N}{d}} .
$$

That is, $S_{N}$ is essentially given by the first term in Eq. (22) and the relative error shrinks with increasing $N$. This is the expected behavior for the dominating state, because $d^{N}\left(1-\frac{1}{d}\right)$ is the absolute maximum the traceless part of a pure state $|\psi\rangle\langle\psi|$ can achieve: An offdiagonal element consisting of orthogonal product states gives $\||j j \ldots j\rangle\langle k k \ldots k| \|_{\mathcal{P}}=1$, which is the maximum among all rank-1 operators. An $N$-qudit state of local dimension $d$ can have at most Schmidt rank $d$ in a bipartition of a single party against the rest. This amounts to a maximum offdiagonal contribution 
of $\frac{1}{d^{2}} d(d-1) d^{N}$ to $S_{N}-$ which is precisely the GHZ result. Consequently, for large $N$ the GHZ state $N$-sector approaches the maximum for any rank-1 operator. Evidently, this discussion cannot exclude the existence of a state that approaches this maximum even faster.

\section{Conclusions}

We have analyzed the Bloch sector distribution for multipartite pure quantum states of $N d$-level systems, in particular the properties of the $N$-sector. We have demonstrated that the sectors must not be considered individually; rather, there are numerous interdependencies that determine the distribution. One of our main results based on this insight is the proof that for qubits the GHZ state has maximum $N$-sector also for even $N$. We have given an extensive characterization of the $N$-sector behavior for arbitrary $N$ and $d$, which can be viewed as an algebraic problem analogous to that of the existence of AME states. Most importantly, we find that strong $N$-party correlations (viz maximum $N$-sector) do not necessarily imply genuine multipartite entanglement. Apart from our physics results, our work provides several novel technical tools for analyzing the Bloch representation of pure states and thereby shows that this is a powerful approach to obtain new insight into the mathematical properties of many-body quantum states.

\section{Acknowledgments}

This work was funded by the German Research Foundation Project EL710/2-1 (C.E., J.S.), by Grant PGC2018-101355-B-100 (MCIU/FEDER/UE) and Basque Government Grant IT986-16 (J.S.). The authors would like to thank Marcus Huber and Nikolai Wyderka for stimulating discussions. C.E. and J.S. acknowledge Klaus Richter's support of this project.

\section{Appendix}

\section{Sector distribution of GHZ state, Eq. (8)}

In order to obtain the sector lengths for the GHZ state it is not necessary to explicitly calculate the
Bloch representation. Yet we quickly do it for the qubit example to demonstrate how simple it is. The density matrix of the $N$-qubit GHZ state is

$$
\begin{aligned}
\Pi_{\mathrm{GHZ}_{2}^{N}}=\frac{1}{2}( & |00 \ldots 0\rangle\langle 00 \ldots 0|+ \\
& +|11 \ldots 1\rangle\langle 11 \ldots 1|+ \\
& +|00 \ldots 0\rangle\langle 11 \ldots 1|+ \\
& +|11 \ldots 1\rangle\langle 00 \ldots 0|) .
\end{aligned}
$$

Each term here is a tensor product of $N$ identical rank-1 single-qubit operators,

$$
\begin{aligned}
|0\rangle\left\langle\left. 0\right|^{\otimes N}\right. & =\frac{1}{2^{N}}(\mathbb{1}+Z)^{\otimes N} \\
|1\rangle\left\langle\left. 1\right|^{\otimes N}\right. & =\frac{1}{2^{N}}(\mathbb{1}-Z)^{\otimes N} \\
|0\rangle\left\langle\left. 1\right|^{\otimes N}\right. & =\frac{1}{2^{N}}(X+\mathrm{i} Y)^{\otimes N} \\
|1\rangle\left\langle\left. 0\right|^{\otimes N}\right. & =\frac{1}{2^{N}}(X-\mathrm{i} Y)^{\otimes N},
\end{aligned}
$$

where $X \equiv \sigma_{1}, Y \equiv \sigma_{2}, Z \equiv \sigma_{3}$ are the Pauli matrices and $\mathbb{1}$ is the qubit identity matrix. Hence

$$
\begin{aligned}
\Pi_{\mathrm{GHZ}_{2}^{N}} & =\frac{1}{2^{N}}\left(\sum_{\text {even\# } Z} Z Z \ldots \mathbb{1}+\right. \\
& \left.+\sum_{\text {even\#Y }}(-1)^{\frac{\# Y}{2}} X \ldots Y Y \ldots X \ldots Y Y\right)
\end{aligned}
$$

where the sums run over all combinations of even numbers of $Z$ occurrences padded with $1 \mathrm{~s}$ (diagonal) and even numbers of $Y$ occurences padded with $X$ s (offdiagonal); for simplicity we omit the tensor product signs. The difference between even and odd $N$ is that for even $N$ there is one $N$-sector term $Z Z \ldots Z$ in the diagonal part, whereas for odd $N$ the $N$-sector exclusively consists of offdiagonal terms. The GHZ state for $d>2$ can be built in an analogous manner.

In order to derive Eq. (8) we can take a shortcut and use Eq. (10),

$$
S_{N}=d^{N} \sum_{A}\left(-\frac{1}{d}\right)^{|A|} \operatorname{Tr}\left(\rho_{\bar{A}}^{2}\right)
$$

The GHZ state is particularly simple as all reduced states are of rank $d$ and completely mixed on their span, so that $\operatorname{Tr}\left(\rho_{A}^{2}\right)=\frac{1}{d}$ for all 
$|A| \neq 0, N$. For $|A|=0$ and $|A|=N$ we have $\operatorname{Tr}\left(\rho_{A}\right)^{2}=1$, so that

$$
\begin{aligned}
S_{N}= & d^{N} \frac{1}{d}\left(1-\frac{1}{d}\right)^{N}+d^{N}\left(1-\frac{1}{d}\right)- \\
& -(-1)^{N} d^{N}\left(\frac{1}{d^{N+1}}-\frac{1}{d^{N}}\right) \\
= & d^{N-1}(d-1)+ \\
& +\frac{1}{d}\left[(d-1)^{N}+(-1)^{N}(d-1)\right] .
\end{aligned}
$$

For $k<N, S_{k}$ is given by the length of the last sector of the reduced density matrix $\rho_{A}$ $(|A|=k)$ times the number of such reduced density matrices, $S_{k}=d^{k}\left(\begin{array}{c}N \\ k\end{array}\right)\left\|\rho_{A}\right\|_{\mathcal{P}}^{2}$. In contrast to the $N$-sector we need not include a correction for the first term, so that

$$
\begin{aligned}
S_{k}= & d^{k} \frac{1}{d}\left(1-\frac{1}{d}\right)^{k}\left(\begin{array}{c}
N \\
k
\end{array}\right)- \\
& -(-1)^{k} d^{k}\left(\frac{1}{d^{k}}-\frac{1}{d^{k-1}}\right) \\
= & \left(\begin{array}{c}
N \\
k
\end{array}\right) \frac{1}{d}\left[(d-1)^{k}+(-1)^{k}(d-1)\right] .
\end{aligned}
$$

\section{Proof maximum $N$ sector of odd- $N$ qubit GHZ state}

Here we show the proof that for odd $N$ qubits, the maximum $N$-sector length is $\max S_{N}=$ $S_{N}(\mathrm{GHZ})=2^{N-1}$, which is realized by the GHZ state [6].

First, we recall that for odd $N$ qubit states $\Pi=|\psi\rangle\langle\psi|$ the degree-2 SL invariant

$$
H=\operatorname{Tr}\left[\Pi Y^{\otimes N} \Pi^{*} Y^{\otimes N}\right]=0
$$

always vanishes $[5,6,10]$ (here, $Y \equiv \sigma_{2}$ is a Pauli matrix and $\Pi^{*}=\left|\psi^{*}\right\rangle\left\langle\psi^{*}\right|$, where $\left|\psi^{*}\right\rangle$ is the vector with complex conjugate components). In terms of sector lengths Eq. (A6) reads $[5,6,10]$ $0=\sum_{k=0}^{N}(-1)^{k} S_{k}$, so that

$$
S_{\text {even }} \equiv \sum_{k=0}^{\frac{N-1}{2}} S_{2 k}=\sum_{k=0}^{\frac{N-1}{2}} S_{2 k+1} \equiv S_{\text {odd }}
$$

that is, the sum of the even-numbered sector lengths $S_{\text {even always equals that of the }}$ odd-numbered ones, $S_{\text {odd }}$. Because of the purity-0 constraint $\sum_{k=0}^{N} S_{k}=S_{\text {even }}+S_{\text {odd }}=2^{N}$ this means that both even and odd sector length sums are always equal to $2^{N-1}$, so that the properties of a state are encoded in the distribution of the even sector lengths among themselves on the one hand, and separately the odd ones, on the other hand.

It is quite obvious then that the GHZ state (odd $N$ ) is the one with maximum $N$-sector: Here, the entire odd sector length $2^{N-1}$ is shifted to the $N$-sector, and the other odd sector lengths vanish. (The peculiarity is that such a state actually does exist - this is by no means guaranteed by Eq. (A7) and the purity constraint.) Note also that for the GHZ state, Eq. (A7) does not say anything about the distribution of the even-numbered sectors.

Now consider the $\mathcal{P} \mathcal{Q}$ relation, Eq. (13) from the main text,

$$
\begin{aligned}
& S_{N}=\frac{1}{2^{N}} \sum_{k=0}^{N}(-1)^{k} 3^{N-k} S_{k} \\
& =\frac{1}{2^{N}}\left[\sum_{l=0}^{\frac{N-1}{2}} 3^{N-2 l} S_{2 l}-\sum_{m=0}^{\frac{N-1}{2}} 3^{N-2 m-1} S_{2 m+1}\right] .
\end{aligned}
$$

We see that also from the point of view of Eq. (A8) the maximum $N$-sector for the GHZ state makes perfect sense: All odd sector contributions are moved to $S_{N}$ where they cause the 'least damage' for maximizing the r.h.s. of the equation, because $S_{N}$ has the smallest prefactor.

\section{Proof maximum I.h.s. of Eq. (18) in main text}

In the following we demonstrate the last step of the proof in the main text that $\max S_{N}=$ $2^{N-1}+1$ for even- $N$ qubit GHZ states. This step consists in maximizing the left-hand side (l.h.s.) of Eq. (18) of the manuscript,

$$
S_{1}+\sum_{j} 2 \operatorname{Tr} R_{[j]} \longrightarrow \max ,
$$

where $\rho_{[j]}=\operatorname{Tr}_{\{j\}} \Pi_{x}$ and $\Pi_{x}=|x\rangle\langle x|$ is a pure state.

First, let us consider $\rho_{[1]}=\operatorname{Tr}_{\{1\}} \Pi_{x}$. We write the Schmidt decomposition of $|x\rangle$ with respect to the first qubit,

$$
|x\rangle=\sqrt{\lambda_{1}}|0\rangle\left|X_{0}\right\rangle+\sqrt{1-\lambda_{1}}|1\rangle\left|X_{1}\right\rangle,
$$

where $\{|0\rangle,|1\rangle\}$ is the Schmidt basis on the first qubit and $\left|X_{0}\right\rangle,\left|X_{1}\right\rangle$ two orthogonal odd- $(N-1)$ 
qubit states, the Schmidt vectors on qubits $\{2 \ldots N\}$. Hence,

$$
\rho_{[1]}=\lambda_{1}\left|X_{0}\right\rangle\left\langle X_{0}\left|+\left(1-\lambda_{1}\right)\right| X_{1}\right\rangle\left\langle X_{1}\right|,
$$

so that, as for $k$-qubit states $|\phi\rangle$ the inverted state $|\tilde{\phi}\rangle=Y^{\otimes k}\left|\phi^{*}\right\rangle[\mathrm{cf} .[5,9,10]$ and the discussion below Eq. (A6)],

$$
\begin{aligned}
\operatorname{Tr} R_{[1]}= & \operatorname{Tr}\left[\rho_{[1]} Y^{\otimes(N-1)} \rho_{[1]}^{*} Y^{\otimes(N-1)}\right] \\
= & \lambda_{1}^{2}\left|\left\langle X_{0} \mid \tilde{X}_{0}\right\rangle\right|^{2}+\left.\left(1-\lambda_{1}\right)^{2}\left\langle X_{1} \mid \tilde{X}_{1}\right\rangle\right|^{2}+ \\
& +\lambda_{1}\left(1-\lambda_{1}\right)\left|\left\langle X_{0} \mid \tilde{X}_{1}\right\rangle\right|^{2}+ \\
& +\lambda_{1}\left(1-\lambda_{1}\right)\left|\left\langle X_{1} \mid \tilde{X}_{0}\right\rangle\right|^{2} \\
= & 2 \lambda_{1}\left(1-\lambda_{1}\right)\left|\left\langle X_{0} \mid \tilde{X}_{1}\right\rangle\right|^{2} \\
= & 2 \lambda_{1}\left(1-\lambda_{1}\right)\left|\left\langle X_{0} \mid Y^{\otimes(N-1)} X_{1}^{*}\right\rangle\right|^{2}
\end{aligned}
$$

because $\langle\phi \mid \tilde{\phi}\rangle=0$ for odd- $N$ qubit states [see also Eq. (A6)]. For the matrix element in Eq. (A11) we have

$$
\Delta=\left|\left\langle X_{0} \mid Y^{\otimes(N-1)} X_{1}^{*}\right\rangle\right| \leqq 1
$$

since the operator $Y^{\otimes(N-1)}$ has only eigenvalues of modulus 1. Consequently we find, if we add the 1 -sector $S_{1}^{(1)}$ of the 1 st qubit in $\Pi_{x}$,

$$
\begin{aligned}
& \max _{|x\rangle}\left[S_{1}^{(1)}+2 \operatorname{Tr} R_{[1]}\right]= \\
& =\max _{\lambda_{1}, \Delta}\left[2 \lambda_{1}^{2}+2\left(1-\lambda_{1}\right)^{2}-1+4 \lambda_{1}\left(1-\lambda_{1}\right) \Delta^{2}\right] \\
& =1 .
\end{aligned}
$$

The states $\left|X_{0}\right\rangle,\left|X_{1}\right\rangle$ that realize this maximum are, e.g., $\left|X_{0}\right\rangle=|0\rangle^{\otimes(N-1)}$ and $\left|X_{1}\right\rangle=|1\rangle^{\otimes(N-1)}$. That is, an even- $N$ qubit state that maximizes the l.h.s. of Eq. (A13) is, e.g., the GHZ state $|x\rangle=$ $\frac{1}{\sqrt{2}}\left(|0\rangle^{\otimes N}+|1\rangle^{\otimes N}\right)$.

The same reasoning as above can be applied for all qubits $j=1 \ldots N$, so that we find for the symmetrized 1.h.s. of Eq. (A13),

$$
\max _{|x\rangle}\left[S_{1}+\sum_{j} 2 \operatorname{Tr} R_{[j]}\right]=N,
$$

with the GHZ state attaining the maximum.

\section{References}

[1] U. Fano, A Stokes-Parameter Technique for the Treatment of Polarization in Quantum Mechanics, Phys. Rev. 93, 121 (1954).
[2] U. Fano, Description of States in Quantum Mechanics by Density Matrix and Operator Techniques, Rev. Mod. Phys. 29, 74 (1957).

[3] G. Mahler and V.A. Weberruß, Quantum Networks, 2nd Edition (Springer, Berlin, 2004).

[4] C. Klöckl and M. Huber, Characterizing multipartite entanglement without shared reference frames, Phys. Rev. A 91, 042339 (2015).

[5] C. Eltschka and J. Siewert, Monogamy equalities for qubit entanglement from Lorentz invariance, Phys. Rev. Lett. 114, 140402 (2015).

[6] M.-C. Tran, B. Dakic, F. Arnault, W. Laskowski, and T. Paterek, Quantum entanglement from random measurements, Phys. Rev. A 94, 042302 (2016).

[7] P. Appel, M. Huber, and C. Klöckl, Monogamy of correlations and entropy inequalities in the Bloch picture, J. Phys. Commun. (2020), doi:10.1088/2399-6528/ab6fb.

[8] F. Huber, O. Gühne, and J. Siewert, Absolutely Maximally Entangled States of Seven Qubits Do Not Exist, Phys. Rev. Lett. 118, 200502 (2017).

[9] C. Eltschka and J. Siewert, Distribution of entanglement and correlations in all finite dimensions, Quantum 2, 64 (2018).

[10] N. Wyderka, F. Huber, and O. Gühne, Constraints on correlations in multiqubit systems, Phys. Rev. A 97, 060101 (2018).

[11] F. Huber, C. Eltschka, J. Siewert, and O. Gühne, Bounds on absolutely maximally entangled states from shadow inequalities, and the quantum MacWilliams identity, J. Phys. A: Math. Theor. 51, 175301 (2018).

[12] C. Eltschka, F. Huber, O. Gühne, and J. Siewert, Exponentially many entanglement and correlation constraints for multipartite quantum states Phys. Rev. A 98, 052317 (2018).

[13] T. Cox and P.C.E. Stamp, Partitioned density matrices and entanglement correlators, Phys. Rev. A 98, 062110 (2018). 
[14] N. Wyderka and O. Gühne, Characterizing quantum states via sector lengths, e-print arXiv:1905.06928 (2019).

[15] C. Eltschka and J. Siewert, Joint Schmidt-type decomposition for two bipartite pure states, Phys. Rev. A 101, 022302 (2020).

[16] J. Schlienz and G. Mahler, Description of entanglement, Phys. Rev. A 52, 4396 (1995).

[17] J. Schlienz and G. Mahler, The maximal entangled three-particle state is unique, Phys. Lett. A 224, 39 (1996).

[18] M. Żukowski and C. Brukner, Bell's theorem for general $N$-qubit states, Phys. Rev. Lett. 88, 210401 (2002).

[19] M. Teodorescu-Frumosu and G. Jaeger, Quantum Lorentz-group invariants of n-qubit systems, Phys. Rev. A 67, 052305 (2003).

[20] H. Aschauer, J. Calsamiglia, M. Hein, and H.J. Briegel, Local invariants for multi-partite entangled states allowing for a simple entanglement criterion, Quantum Inf. Comput. 4, 383 (2004); journal link; arXiv.org link.

[21] A. J. Scott, Multipartite entanglement, quantum error correcting codes, and entangling power of quantum evolutions, Phys. Rev. A 69, 052330 (2004).

[22] J.I. de Vicente, Separability criteria based on the Bloch representation of density matrices, Quantum Inf. Comput. 7, 624 (2007); journal link; arXiv.org link.

[23] J.I. de Vicente, Further results on entanglement detection and quantification from the correlation matrix criterion, J. Phys. A: Math. Theor. 41, 065309 (2008).

[24] P. Badziag, C. Brukner, W. Laskowski, T. Paterek, and M. Żukowski, Experimentally Friendly Geometrical Criteria for Entanglement, Phys. Rev. Lett. 100, 140403 (2008).

[25] W. Laskowski, M. Markiewicz, T. Paterek, and M. Żukowski, Correlation-tensor criteria for genuine multiqubit entanglement, Phys. Rev. A 84, 062305 (2011).
[26] J.I. de Vicente and M. Huber, Multipartite entanglement detection from correlation tensors, Phys. Rev. A 84, 062306 (2011).

[27] We will use the term " $k$-sector length" instead of "squared $k$-sector length" following Ref. [6]. In the present context this does not lead to confusion.

[28] One may imagine very different correlation quantifiers, e.g., D. Girolami, T. Tufarelli, and C.E. Susa, Quantifying Genuine Multipartite Correlations and their Pattern Complexity, Phys. Rev. Lett. 119, 140505 (2017).

[29] J. Kaszlikowski, A. Sen De, U. Sen, V. Vedral, A. Winter, Quantum Correlation Without Classical Correlations, Phys. Rev. Lett. 101, 070502 (2008).

[30] C. Schwemmer, L. Knips, M.C. Tran, A. de Rosier, W. Laskowski, T. Paterek, and H. Weinfurter, Genuine Multipartite Entanglement without Multipartite Correlations, Phys. Rev. Lett. 114, 180501 (2015).

[31] M.C. Tran, M. Zuppardo, A. de Rosier, L. Knips, W. Laskowski, T. Paterek, and $\mathrm{H}$. Weinfurter, Genuine $N$-partite entanglement without $N$-partite correlation functions, Phys. Rev. A 95, 062331 (2017).

[32] W. Klobus, W. Laskowski, T. Paterek, M. Wiesniak, and H. Weinfurter, Higher dimensional entanglement without correlations, Eur. Phys. J. D 73, 29 (2019).

[33] This relation corresponds to a special case of the quantum MacWilliams identity, cf. Ref. [11].

[34] V. Coffman, J. Kundu, and W.K. Wootters, Distributed entanglement, Phys. Rev. A 61, 052306 (2000).

[35] P. Rungta, V. Buzek, C.M. Caves, M. Hillery, and G.J. Milburn, Universal state inversion and concurrence in arbitrary dimensions, Phys. Rev. A 64, 042315 (2001).

[36] W. Hall, Multipartite reduction criteria for separability, Phys. Rev. A 72, 022311 (2005).

[37] M. Lewenstein, R. Augusiak, D. Chruściński, S. Rana, and J. Samsonowicz, Sufficient separability criteria and linear maps, Phys. Rev. A 93, 042335 (2016). 
[38] An in-depth analysis of this projection operator will be carried out in forthcoming work.

[39] D. Goyeneche and K. Życzkowski, Genuinely multipartite entangled states and orthogonal arrays, Phys. Rev. A 90, 022316 (2014).

[40] D. Goyeneche, D. Alsina, J.I. Latorre, A. Riera, and K. Życzkowski, Absolutely maximally entangled states, combinatorial designs, and multiunitary matrices, Phys. Rev. A 92, 032316 (2015). 